\title{
The Eurocentrism of Pathological Eponyms
}

\author{
Karthyayani Priya Satish ${ }^{1}$ (1)
}

Accepted: 7 January 2022 / Published online: 21 January 2022

(c) The Author(s) under exclusive licence to International Association of Medical Science Educators 2022

\section{Dear Editor,}

I read the publication entitled 'Ten Global Challenges in Medical Education: Wicked Issues and Options for Action' by Stewart Mennin (Medical Science Educator, September 20, 2021), with great interest [1]. The author is congratulated for dissecting the hidden challenges that confront effective medical education, especially the wicked issues. The suggestions provided to tame the wicked issues, in particular the 'inquiry', are fascinating and can be applied to everyday issues faced by medical students. Indeed, self-reflection, shared exploration, and turning assumptions into questions have inadvertently evaporated from medical education.

Taking a cue from Mennin's article, I wish to bring to the attention of the leaders of medical education the unique challenges faced by international medical students. The pandemic-driven structural changes to the sudden transition to online, contactless learning have disrupted the traditional learning systems prevalent in medical colleges in India. Almost all areas of medicine rely on using relational cues and metaphorical descriptions to recognize patterns, make diagnoses, and simplify complex concepts to enhance understanding by medical students, particularly pathology [2]. However, the concepts currently employed to serve this purpose may fall short in a global medical curriculum composed of international medical students. This is particularly true in pathology, where the individual student may have little or no prior exposure to eponymophilia [3]. Eponymophilia is derived from the use of eponyms by pathologists to understand complex patterns more comfortably. Learning and practicing relational cues and metaphorical descriptions are postulated to enhance the development of visual intelligence in diagnosing towards the provision of quality patient care [4].

Karthyayani Priya Satish

kartfamily2020@gmail.com

1 Kasturba Medical College, Karnataka, Mangalore 575001, India
Vimal and Nishanti have compiled a comprehensive list of the eponyms used in pathology including coffee-ground emesis, strawberry tongue, and rice-water stools $[5,6]$. Descriptors of disease are crammed into catchy buzzwords, most of which can be found in a common Western refrigerator. Unfortunately, the efficacy of such descriptors wanes the further removed a student finds themselves from the West; pathology is, essentially, a Eurocentric subject. Some eponyms are inaccessible due to pure obscurity; the 'anchovy sauce' in anchovy sauce-pus is a head-scratcher in a culture where the condiment is in short supply. The famous 'strawberry tongue', a marker of Kawasaki disease, loses its purpose in a rural setting where strawberries are as hard to come by as unicorns. Other eponyms are hindered by the turns of the language they employ; while most medical students have, it could be argued, eaten an orange at some point in their life, the descriptor 'peau d'orange' - used to indicate a dimpled, orange-skin appearance in breast cancer-finds itself lost in translation. Similarly, the term 'sago spleen' brings about great confusion when discussed in a lecture, but to asterisk it with 'sabudana', the Indian equivalent of the term, will have a non-Western class feeling much more comfortable with the term.

While it may be suggested that the advent of the Internet has made even the most obscure of terms searchable, access to the Internet in developing nations is not always a guarantee, and students on finding unfamiliar terminology may resort to rote memorization rather than attempts at understanding. If the epidemiology data in textbooks can vary according to the country from where it is published, the frequent use of the asterisk becomes feasible, if not crucial. The simple inclusion of 'meduvada' beside 'anastomotic doughnut' in an Indian pathology textbook would be a small step towards creating a more inclusive, globally centred pathology curriculum.

Given all the challenges for medical education, particularly for global medical students, it is no surprise that the 11th challenge is the Eurocentrism of eponyms in pathology, in addition to the 'ten challenges and wicked issues' discussed by Mennin [1]. 


\section{Declarations}

Conflict of Interest The author declares no competing interests.

\section{References}

1. Mennin S. Ten global challenges in medical education: wicked issues and options for action. Medical Science Educator. 2021.

2. Masukume G, Zumla A. Analogies and metaphors in clinical medicine. Clin Med (Lond). 2012;12(1):55-6. https://doi.org/10.7861/ clinmedicine.12-1-55.PMID:22372224;PMCID:PMC4953421.

3. Ahmed H, Ogala WN, Ibrahim M. Culinary metaphors in Western medicine: a dilemma of medical students in Africa. Med Educ. 1992;26(5):423-4. https://doi.org/10.1111/j.1365-2923.1992. tb00195.x (PMID: 1435383).
4. Bastardot F, Gachoud D. Le diagnostic visuel: au carrefour de la pédagogie médicale et des progrès en intelligence artificielle [Visual diagnosis: between medical education and advances in artificial intelligence]. Rev Med Suisse. 2019;15(672):2145-2149. French. (PMID: 31746571).

5. Vimal M, Nishanthi A. Food eponyms in Pathology. Journal of Clinical and Diagnostic Research: JCDR. 2017;11(8):EE01. https:// doi.org/10.7860/JCDR/2017/28375/10360.

6. Gishen F, Lokugamage A. Diversifying and decolonising the medical curriculum. 2018 BMJ. https://blogs.bmj.com/bmj/2018/10/ 18/diversifying-and-decolonising-the-medical-curriculum/.

Publisher's Note Springer Nature remains neutral with regard to jurisdictional claims in published maps and institutional affiliations. 